\title{
International Comparison Analysis of the Value Added Share of Imported Intermediate Goods: Based on Non-Competitive Input-Output Model
}

\author{
Huang Chengcheng \\ School of Economics, Shanghai University, Shanghai, China \\ Email address: \\ hwangzeng@163.com
}

\section{To cite this article:}

Huang Chengcheng. International Comparison Analysis of the Value Added Share of Imported Intermediate Goods: Based on Non-Competitive Input-Output Model. Science Innovation. Vol. 4, No. 3, 2016, pp. 142-145. doi: 10.11648/j.si.20160403.14

Received: April 13, 2016; Accepted: May 11, 2016; Published: June 8, 2016

\begin{abstract}
In this paper, we use the input-output model to calculate each sectors' value added share of imported intermediate goods via eight representative countries' input-output tables, to explore the relation between each sectors' value added share of imported intermediate goods and the specialty industries of each countries. The analysis results show that there is a positive correlation among one country's specialty industry and the value added share of imported intermediate goods of its upstream industry.
\end{abstract}

Keywords: Input-output Model, Imported Intermediate Goods, Value Added Share, Upstream Sector

\section{进口中间品增加值比重的国际比较分析： 基于非竞争性投入产出模型}

\section{黄诚诚}

经济学院, 上海大学, 上海, 中国

\section{邮箱}

hwangzeng@163.com

摘要: 本文利用投入产出模型计算出各部门的进口中间品增加值比重, 来探索部门间进口中间品比重的大小和该国优 势产业的联系，分析结果表明，一国优势产业应该和该产业上游产业的进口中间品的比重大小有着正相关关系。

关键词：投入产出模型，进口中间品，增加值比重，上游部门

\section{1. 引言}

随着全球化程度不断加深, 对于中间品贸易及其增加 值的研究重要性愈发凸显。一般来说, 早期中国的出口产 业主要体现为劳动密集型, 近年来这种生产、贸易模式逐 渐不能很好地适应新的经济发展要求, 其表现在于: 一方
面, 中国和贸易伙伴国往往维持着巨额贸易顺差, 这为中 国带来巨大财富的同时也常导致贸易利益分配的争议; 另 一方面, 中国的贸易出口增加值之中很大比例实际上来自 进口中间产品, 中国本身生产的增加值相对有限。

根据上述背景，本文关注问题是：（1）一个国家各 个部门出口增加值与其本部门或者相关部门进口中间产 
品比重关系; (2) 在经济规模不同的国家进口中间品增 加值比重分布差别。

对上述问题思考采取的研究方法是：首先对中国、美 国、日本、德国、英国、印度、俄罗斯、巴西8个国家投 入产出表进行计算分析, 然后先探究中国1995年以来几个 重要产业部门的进口中间品增加值比重变化和中国经济 发展的关系, 再去比较不同国家产业部门间的进口中间品 增加值比重, 来分析这种增加值比重分布差异和该国经济 模式的关联, 最后得出相关的结论, 希望可以为中国的对 外贸易和产业扶持的政策制定工作提供一些参考。

\section{2. 文献综述}

\section{1. 以增加值方法测量贸易利益分配}

早期衡量国际贸易贡献度以及利益分配的标准是统 计贸易顺差（曾铮，张路路 [1]），但是该方法不仅会高 估出口规模总量, 还会对各行业出口规模扭曲 (陈雯, 李 强 [2]）。中国在近几十年的国际贸易中往往被指责贸易 逆差过大, 是国际贸易的利益主要获得者。新的方法是通 过测算中间产品的增加值来衡量贸易的利益分配, 按照这 种算法, 中国对外贸易的差额被高估了差不多 20 40\%（康 振宁, 陈海啸 [3]），中欧贸易（高运胜, 甄程成, 郑乐 凯 [4]）、中日贸易（康振宁, 徐鹏 [5]）以及中美贸易（王 岗, 盛斌 [6]) 都是如此。

\section{2. 中国出口增加值研究现状}

目前学界对于中国出口的增加值的研究指出：（1） 中国在全球价值链中居于低端位置, 垂直专业化水平较低。 尽管中国通过产出供给和投入需求两个渠道深入融入全 球价值链（程大中 [7]）, 但高敬峰, 张艳华 [8]认为中国 对高技术含量的中间产品进口依赖较大，而本国的投入则 多为中低技能劳动。其中, 来自日韩的中间产品增加值占 了约1/3（平新乔等 [9]）。（2）中国对外出口的增加值 来自进口中间产品的比重偏大, 与欧美发达国家相比, 出 口特点是国外增加值较高、国内增加值较低、服务增加值 偏低（廖泽芳, 宁凌 $[10]$ ) 。（3）出口增加值短期提升 较难 (江希, 刘似臣 [11]), 要促进增加值贸易转型升级, 减轻要素流向扭曲, 关键是国内要素禀赋结构优化升级 （程大中 [12]）。

上述的研究显示目前学界关于中国出口增加值的研 究大都集中于贸易利得分配问题, 局限于中国整体进口中 间品对出口增加值的影响, 很少对各个部门进口中间品增 加值比重进行观察分析, 并做出国际比较, 而这即是本文 的研究目的。

\section{3. 理论模型及数据来源}

\section{1. 理论模型}

Humme1s [13] 提出垂直专业化模型, 用以测量 $\mathrm{n}$ 个部门 间进口中间产品对出口增加值的贡献程度:

$$
V S=\sum_{i=1}^{n} V S_{i}=\sum_{i=1}^{n}\left(\frac{M_{i} X_{i}}{Y_{i}}\right)
$$

VS表示垂直专业化程度, $M_{i}$ 是 $\mathrm{i}$ 部门的进口中间产品, $Y_{i}$ 是 $\mathrm{i}$ 部门的产出, $X_{i}$ 是 $\mathrm{i}$ 部门出口值, $\frac{M_{i}}{Y_{i}}$ 表示 $\mathrm{i}$ 部门单位产 出所需要的进口投入比例。

陈宏易 [14] 在此基础上, 考虑到进口中间品在国内各 个部门多阶段循环使用, 修正 (1) 式得到:

$$
V S^{M}=\frac{V S}{X}=\left(\frac{1}{X}\right)\left[u A^{M}\left(I-A^{D}\right)^{-1} x\right]
$$

I是单位矩阵, $A^{D}$ 是 $\mathrm{n} \times \mathrm{n}$ 的生产投入系数矩阵, 那么 $\left(I-A^{D}\right)^{-1}$ 就反映了进口中间产品在国内部门间循环的产 业关联程度, 即波及效应。然后将 $\left(I-A^{D}\right)^{-1}$ 展开为 $I+\left(A^{D}\right)^{1}+\left(A^{D}\right)^{2}+\cdots+\left(A^{D}\right)^{k}$ ( k表示循环的次数), 可 将式(2) 写作:

$$
V S^{M}=V S^{N}+u A^{M}\left[\left(A^{D}\right)^{1} x+\left(A^{D}\right)^{2} x+\cdots+\left(A^{D}\right)^{k} x\right]
$$

如上文所述, $\mathrm{VS}^{M}$ 表示通过多阶段投入产出循环的垂 直专业化出口值, 而 $\mathrm{VS}^{N}$ 表示未通过多阶段投入产出循环 的垂直专业化出口值, 两者相减得：

$$
V S^{V A}=u A^{M}\left[\left(A^{D}\right)^{1} x+\left(A^{D}\right)^{2} x+\cdots+\left(A^{D}\right)^{k} x\right]
$$

$\mathrm{VS}^{V A}$ 就是反映进口中间产品通过国内多阶段投入产 出循环创造的增加值。

\section{2. 数据来源}

本文中使用美国、日本、德国、英国、中国、印度、 俄罗斯、巴西8个国家投入产出表, 时间跨度为1995 2011 年，数据来自世界投入产出数据库（WIOD）。

\section{4. 增加值比重的比较分析}

\section{1. 中国进口中间品VA比重变化分析}

改革开放之后，中国经济规模迅速，这和开展大规模 的对外贸易是分不开的。本文使用的投入产出表的时间段 即对应于该时期。

通过分析投入产出表，可以发现1995 2011年中国的 进口中间品VA比重高的前 5 个部门的比重在这 17 年间发生 了较大变化, 具体如下:

纺织业进口中间品VA比重一路走低, 这说明中国的产 业特征逐渐由纺织品依赖较高的劳动密集型转向资本密 集型和技术密集型。与纺织业相反, 中国采矿业进口中间 品VA比重一路走高无其是 2002 年左右, 其VA比重大的幅度 增加, 其原因就在于中国产能巨大, 需要大量进口资源。 该趋势同一时期在其他国家也存在, 这反应了重工业发展 对其上游部门采矿业的依赖。

电气光学设备部门的进口中间品VA比重在1997年之 后不断增长, 然后在 2002 年左右开始低幅度下降。这显示 了进入WT0后, 发达国家逐渐把电子工业转移到中国, 然 后加工制造业促进中国本土技术进步, 本国中间产品逐步 替代部分外国进口中间产品。类似的, 化工业的进口中间 
品VA比重在1998年之后就不断下降, 这显示了发达国家对 中国的大规模产业转移, 部分替代了直接贸易。

中国的治金业进口中间品VA比重下跌趋势明显。这和 其上游产业采矿业的进口中间品VA比重一直上涨是有关 的，显示了中国冶金业巨大发展甚至于产能过剩。

\section{2. 各国进口中间品VA比重比较分析}

通过陈宏易 [14]的方法，可以计算出上述 8 个国家的 出口产品中进口中间品的VA比重分布。为了方便表述, 这 些国家可以分为 “发达国家” 和 “金砖国家” 两组, 如表 1所示:

表1 各国三大产业进口中间品VA比重（百分比）。

\begin{tabular}{lllll}
\hline & & & \\
\cline { 3 - 4 } & & 三大产业 & 农业 & 服务业 \\
\hline \multirow{4}{*}{ 发达国家 } & 美国 & 1.57 & 82.58 & 15.85 \\
& 日本 & 0.98 & 83.30 & 15.72 \\
& 德国 & 1.19 & 76.84 & 21.98 \\
& 英国 & 1.08 & 65.03 & 33.88 \\
中金砖国家 & 中国 & 3.36 & 87.07 & 9.57 \\
& 巴西 & 2.10 & 78.78 & 19.12 \\
& E\zh10度 & 1.06 & 90.44 & 8.50 \\
\hline
\end{tabular}

注：表1中数据都是2007 2011五年的平均值；德、英两国的三大产业比重之和因为误差积累，不等于100\%，因不影响分析，故不做误差调整。

表1显示，在第一、第二产业上，发达国家进口中间 品VA比重相对金砖国家较低，其农业和制造业进口中间品 VA比重较低是因为服务业的进口中间品VA占的比重更大, 金砖国家反之。中国农业和制造业进口中间品VA数值和比 重都很高, 原因是（1）中国工业化造成本国农业产量下 降, 逐渐依赖进口; (2) 中国制造业生产规模较大且国 际贸易参与度较高。

第三产业上, 发达国家进口中间产品VA无论数值还是 比重都高于金砖国家。金砖国家中巴西的VA比重比较高, 为 $19.12 \%$, 这是因为其服务业比较发达, 对外国服务业进 口较大; 发达国家的服务业产值较大, 人力成本较高, 倾 向于向中印等金砖国家外包, 比如印度的电话呼叫服务, 软件业等。

根据表2数据对各国进口中间品VA比重特征进行分析 如下:

表2 各国进口中间品VA比重最高前三部门（百分比）。

\begin{tabular}{|c|c|c|c|c|}
\hline & & \multicolumn{3}{|l|}{ 部门 } \\
\hline & & VA比重第一高部门 & VA比重第二高部门 & VA比重第三高部门 \\
\hline \multirow{4}{*}{ 发达国家 } & 美国 & 采矿业, $\quad 26.41$ & 冶金业, 15.04 & 化工业, 11.04 \\
\hline & 日本 & 采矿业, 39.70 & 冶金业， 13.42 & 电光设备, 8.65 \\
\hline & 德国 & 冶金业, & 电光设备, 9.77 & 采矿业, $\quad 8.70$ \\
\hline & 英国 & 采矿业, & 机器租赁, 13.03 & 冶金业， 12.12 \\
\hline \multirow{4}{*}{ 金砖国家 } & 中国 & 采矿业, 26. 37 & 电光设备, 24.78 & 化工业, \\
\hline & 巴西 & 化工业, & 采矿业, $\quad 19.90$ & 冶金业, \\
\hline & 印度 & 采矿业, 26. 18 & 回收业, & 冶金业， 15.55 \\
\hline & 俄罗斯 & 交通设备， 24.45 & 化工业, & 机械制造， 12.60 \\
\hline
\end{tabular}

注: 表2中数据是2007 2011五年的平均值。

中、美两国工业规模巨大, 采矿业十分依赖进口原料, 所以采矿业中间产品VA比重较高, 此外VA比重较高的是冶 金业、化工业和电气光学设备, 这是典型发达工业国家特 征。

日本的采矿业中间产品VA比重更高, 为 $39.70 \%$, 因为 岛国缺乏资源, 严重依赖进口; 同时, 日本冶金业和汽车 制造业等重工业发达，作为相关产业必然引起采矿业中间 产品VA走高。日本电气光学设备进口中间品VA比重为 $8.65 \%$, 在发达国家中较低。根据数据, 日本的电气光学 设备进口中间品VA比重在1995年开始上升, 到2000年达到 峰值, 之后开始较大幅度的下降, 只有在2009年和 2011 年略有上升, 这应该和日本的电子产业的中间产品主要在 本国生产消费有很大关系, 反映了日本 “扩大内需” 经济 政策。
德国进口中间品VA分布特点是比重最高的部门是冶 金业, 为 $21.82 \%$, 紧随其后的是电子光学设备 (9.77\%) 和采矿业 (8.70\%)。从这里可以看出德国减少了初级材 料的制造，专注于高级机械工具的生产。

英国机器租赁 (服务业) 进口中间品VA比重较高, 相 对于其他七国, 其采矿业和冶金业中间产品VA比重也较低, 仅为 $14.25 \%$ 和 $12.12 \%$, 这是因为英国注重服务业发展, 逐 渐把制造业转移出去。租赁和商业服务业较高, 更充分显 示了英国对于重型机械以租赁代替制造的经济特征。

巴西农林业发达，但是其自身的化工业难以满足农林 业生产需求, 需要依赖外国农药、化肥等农业化工产品的 大量输入, 所以该国的进口中间品VA比重最大的部门是化 工业。 
印度的制造业相对不发达，所以回收业进口中间品VA 比重较大，许多回收产品被本国重新使用，比如汽车等。

俄罗斯进口中间品VA比重高的部门是交通设备、化工 业和机械。俄罗斯矿产资源丰富, 采矿业的进口中间品VA 比重较低。但是交通设备和机械制造业对外依赖加大，这 两个部门都是和采矿业关联性很强的部门。

\section{5. 结论和建议}

通过上述对比分析发现: 一国比较有代表性的、发展 强势的产业部门, 其相关产业部门尤其是上游产业部门的 进口中间品VA的比重往往比较高, 比较明显的例子如俄罗 斯的优势产业是采矿业, 所以其机械和交通设备部门的进 口中间品VA比重高。该结论给出新的产业扶持思路, 即扶 植手段不仅限于对该产业本身的开放, 还可以对其上游产 业的开放来促进其发展，这对于幼稚产业的保护政策有更 多的参考意义。一般而言, 一国对其幼稚产业的开放是循 序渐进的, 过程可能比较漫长。本文分析的结果指出, 可 以通过早一步对幼稚产业的上游产业的进行, “催熟” 该 产业本身。

\section{致谢}

本文的数据整理得到了沈华夏同学的帮助, 在此致谢。

\section{参考文献}

１１］曾铮, 张路路. 全球生产网络体系下中美贸易利益分配的界 定一一基于中国制造业贸易增加值的研究 $[J]$. 世界经济研 究, 2008, (1) : 36-43。

[2] 陈雯, 李强. 全球价值链分工下我国出口规模的透视分析一 一基于增加值贸易核算方法 $[J]$. 财贸经济， 2014，（7）： $107-135$ 。
[3] 康振宇, 陈海啸. 中国出口贸易的增加值分析 [J]. 中国物价, 2014, (12) : 18-21。

[4] 高运胜, 甄程成, 郑乐凯. 中国制成品出口欧盟增加值分解 研究一一基于垂直专业化分工的视角 $[\mathrm{J}]$. 数量经济技术经 济研究, 2015, (9)：73-88。

[5] 康振宇, 徐鹏. 全球价值链时代的中日贸易分析一一基于增 加值的视角 $[J]$. 国际贸易问题，2015，(4)：75-84。

[6] 王岗, 盛斌. 全球价值链分工背景下的中美增加值贸易与双 边贸易利益 $[J]$. 财经研究， 2014，（9）：97-108。

[7] 程大中. 中国参与全球价值链分工的程度及演变趋势-一 基于跨国投入一产出分析 $[J]$. 经济研究, 2015, (9): 4-16。

[8］高敬峰, 张艳华. 中国出口中的国内增加值与要素报酬解构 [J]. 世界经济研究, 2014, (7) : 22-27。

[9] 平新乔, 郝朝艳, 毛亮, 李化松, 张璐, 胡向婷. 中国出口 贸易中的垂直专门化与中美贸易 [J]. 世界经济, 2006, (5) : $6-10$ 。

[10］廖泽芳, 宁凌. 中国的全球价值链地位考察——基于附加值 贸易视角 $[J]$. 国际商务，2013，(6)：21-30。

[11] 江希, 刘似臣. 中国制造业出口增加值及影响因素的实证研 究——以中美贸易为例 $[J]$. 国际贸易问题, 2014, (11) : 89-98。

[12] 程大中. 中国增加值贸易隐含的要素流向扭曲程度分析 [J]. 经济研究，2014，（9）：105-120。

[13] D. Hummels, Jun Ishii, Kei-Mu Yi. The Nature and Growth of Vertical Specialization in World Trade[J]. Journal of International Economics, 2001, 54(1) : 75-96.

[14] 陈宏易. 从国际垂直分工的观点探讨台湾贸易的形态及其 变动 $[J]$. 台湾经济预测与政策, 2002, 32(2)：60-92。 